SPIE Optics and Photonics Conference, August 10-14, 2008, San Diego, CA

\title{
IR spectrometer using 90-degree off-axis parabolic mirrors
}

\author{
Robert M. Malone \\ National Security Technologies, LLC, Los Alamos Operations \\ Ian J. McKenna \\ National Security Technologies, LLC, Special Technologies Laboratory \\ Daniel H. Dolan \\ Sandia National Laboratories
}

\begin{abstract}
A gated spectrometer has been designed for real-time, pulsed infrared (IR) studies at the National Synchrotron Light Source at the Brookhaven National Laboratory. A pair of 90-degree, off-axis parabolic mirrors are used to relay the light from an entrance slit to an output recording camera. With an initial wavelength range of 1500-4500 nm required, gratings could not be used in the spectrometer because grating orders would overlap. A magnesium oxide prism, placed between these parabolic mirrors, serves as the dispersion element. The spectrometer is doubly telecentric. With proper choice of the air spacing between the prism and the second parabolic mirror, any spectral region of interest within the InSb camera array's sensitivity region can be recorded. The wavelengths leaving the second parabolic mirror are collimated, thereby relaxing the camera positioning tolerance. To set up the instrument, two different wavelength (visible) lasers are introduced at the entrance slit and made collinear with the optical axis via flip mirrors. After dispersion by the prism, these two laser beams are directed to tick marks located on the outside housing of the gated IR camera. This provides first-order wavelength calibration for the instrument. Light that is reflected off the front prism face is coupled into a high-speed detector to verify steady radiance during the gated spectral imaging. Alignment features include tick marks on the prism and parabolic mirrors. This instrument was designed to complement single-point pyrometry, which provides continuous time histories of a small collection of spots from shock-heated targets.
\end{abstract}

Keywords: mid-IR imaging, IR spectrometer, off-axis parabolic mirrors, IR camera, Brookhaven National Synchrotron Light Source

This work was done by National Security Technologies, LLC, under Contract No. DE-AC52-06NA25946 with the U.S. Department of Energy. Sandia National Laboratories operates for the National Nuclear Security Administration under contract DE-AC04-94AL8500. 